\title{
Impacts and effects of COVID-19 infection in pregnancy
}

Amala Sunder, MRCPI (OBG), Bessy Varghese, DGO, Basma Darwish, MD, Noor Shaikho, MD, BCh BAO, Mooza Rashid, FRCOG.

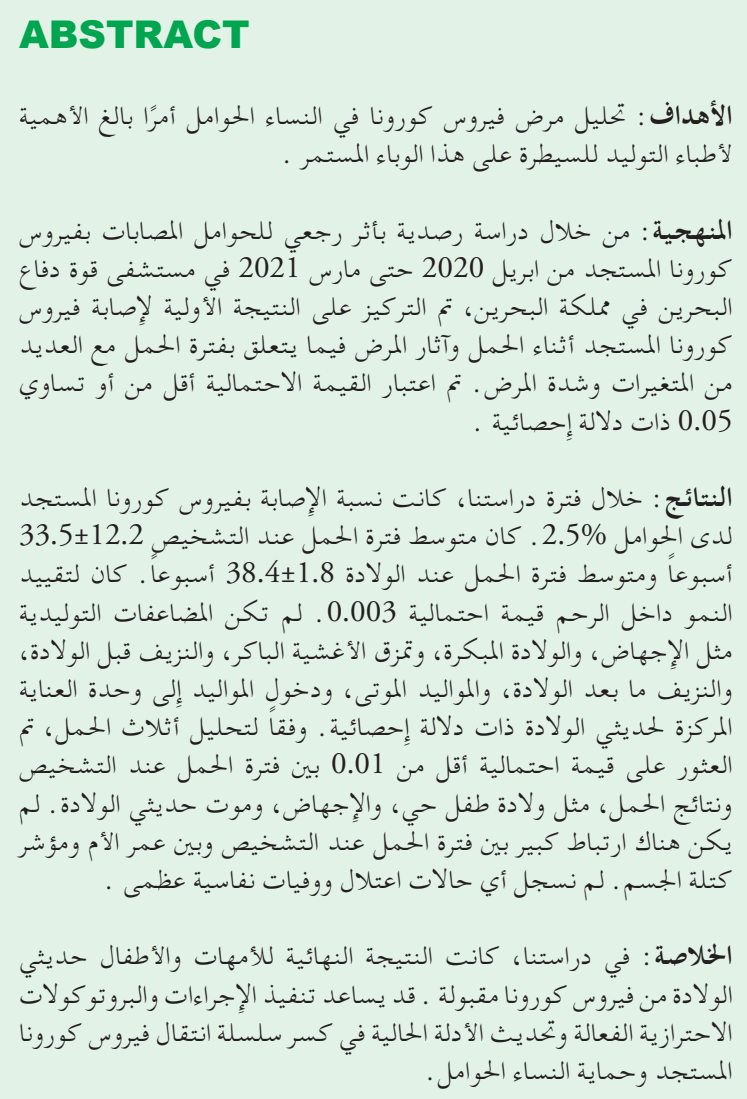

Objectives: To explore the trimester wise significance of the primary outcome in pregnant women during coronavirus disease-19 (COVID-19) pandemic.

Methods: Retrospective observational study of pregnant women who were infected with COVID-19 from April 2020 until March 2021 at Bahrain Defense Force Hospital, Riffa, Bahrain. The study focused on the effects in relation to gestational age (GA), association with variables, severity, and treatment. A $p$-value of $\leq 0.05$ was considered significant.

Results: During the study period, 74 COVID-19 cases were identified from the recorded 2944 pregnant

women. The mean GA at diagnosis was $33.5 \pm 12.2$ weeks, and the mean GA at birth was $38.4 \pm 1.8$ weeks.
Analysis of the obstetric complications revealed fetal growth restriction (FGR) had a $p$-value of $<0.001$. According to the trimester wise analysis, between the gestational period at diagnosis and the outcome of pregnancy, significant $p$-value of $<0.01$ was found in miscarriage. There were no significant associations found in GA at diagnosis and delivery, complications in relation to maternal age and body mass index, and no maternal morbidities or mortalities.

Conclusion: In our study, FGR and miscarriage were the identified complications. However, the maternal and neonatal end result of COVID-19 was satisfactory.

Keywords: fetal growth restriction, miscarriage, stillborn, gestational age, mortality

Saudi Med J 2022; Vol. 43 (1): 67-74 doi: 10.15537/smj.2022.43.1.20210694

From the Department of Obstetrics and Gynaecology, Bahrain Defence Force Hospital, Riffa, Bahrain.

Received 30th August 2021. Accepted 23rd November 2021.

Address correspondence and reprint request to: Dr. Amala Sunder, Department of Obstetrics and Gynaecology, Bahrain Defence Force Hospital, Riffa, Bahrain.E-mail: sunderamala1@yahoo.co.in ORCID ID: https://orcid.org/0000-0003-2314-3592

Coronavirus disease-19 (COVID-19) is a pandemic since December 2019. ${ }^{1}$ Severe acute respiratory syndrome coronavirus-2 (SARS-CoV-2) strain had been first identified in the Wuhan city in China. ${ }^{2}$ Since then, different variant strains have been recognized globally. The majority of these mutations are clinically less considerable. The Middle East respiratory syndrome (MERS-CoV), the South African variant, SARS-CoV, the double mutant variant (B.1.617) in India, P.I, the Brazilian variant (CAL.20C), the United States of America variant (B.1.1.7), the variant from the United Kingdom, and the delta plus and lambda variant, all created major clinical crisis. Human Coronavirus 229E, NL63, OC43, and HKU1 are linked with the varying 
respiratory symptoms. ${ }^{1}$ More new variants of the virus are anticipated. The course of these new variants is not clear. Future studies are needed to clarify whether these variants remain unchanged or keep on mutating or dissipating from the globe as it created a global crisis. ${ }^{2,3}$

Various studies showed that the virus has potential effects on the neurological and respiratory systems due to the cytokine storm and immune reaction in pregnancy. ${ }^{4-6}$ The complexity of the disease was found more in women with maternal age more than 35 . The comorbidities included obesity, diabetes mellitus (DM), hypertension, chronic lung disease, chronic heart disease, renal disorders, blood disorders, malignancies, and women with immunocompromised medications. ${ }^{6,7}$ In general, pregnant women with the disease symptoms are associated with severity and intensive care unit (ICU) admissions compared with non-pregnant. Their existence are more in the third trimester. ${ }^{1,7}$ We analyzed the prognosis and outcome of the disease according to the gestational age (GA) in our pregnant population. However, most of the studies highlighted no difference in complications during pregnancy while comparing with the general population. ${ }^{8}$ Potential adverse effects of the disease in pregnancy includes: venous thromboembolism, acute respiratory distress syndrome, ICU admission, pneumonia, sepsis, thrombocytopenia, viral myocarditis, cardiomyopathy, mortality, increased cesarean rate, miscarriage, preeclampsia, preterm labour, fetal growth restriction (FGR), stillborn, neonatal morbidities and mortalities. ${ }^{1,6,8}$

The first confirmed case in Bahrain was in February 2020. the Royal Bahrain Defense Force Hospital is the tertiary center. It has a 400-bed capacity and is one of the major hospitals in Bahrain. The National Taskforce team made challenging efforts to minimize the transmission of the virus, essential steps regarding health measures and quarantine facilities without any impediment.

The nation has promptly introduced favorable protocols to citizens and residents to combat the pandemic. The Royal Bahrain Defense Force Hospital, Riffa, Bahrain, is one of the dedicated COVID-19 unit that has a fully functional field ICU equipped with modern critical care facilities and each bed is equipped with a ventilator. During COVID-19 pandemic, the maternity unit established a committed team and isolated ward to impart the service.

Disclosure. Authors have no conflict of interests, and the work was not supported or funded by any drug company.
Methods. This is a retrospective observational study of COVID-19 positive pregnant women at Royal Bahrain Defense Force Hospital, Riffa, Bahrain, for 12 months from April 2020 until March 2021.

The inclusion criteria are confirmed cases of disease existence during pregnancy at any gestational period or at the time of labour. Women who were diagnosed COVID-19 positive before pregnancy and after delivery were excluded from the study. The diagnosis was made from the nasopharyngeal samples using polymerase chain reaction (PCR) or SARS-CoV-2 GeneXpert.

The study design was approved by the Ethical Committee Research Centre in Bahrain Defense Force Hospital, Riffa, Bahrain, and The national COVID-19 clinical research team. All data included are anonymized. Medical records were reviewed with confidentiality. The data included demographic data, maternal characteristics, comorbidities, clinical presentation, treatment details, and obstetric outcome. We looked at the association between the GA at diagnosis and at delivery, obstetric complications in relation with gestational age and the complications were compared with the COVID-19 negative group during the same period of study.

Statistical analysis. Continuous variables were constituted as mean and standard deviation. Categorical variables were added as frequencies and percentages. To compare between COVID-19 positive and COVID-19 negative groups, either Chi-square or Fishers exact test were used. Association between variables was evaluated with Pearson correlation coefficient, Spearman's rank correlation coefficient, Chi-square test of independence, and Fisher's exact test as appropriate. A $p$-value of $\leq 0.05$ was considered significant. Statistical Package for the Social Sciences, version 25 (IBM Corp., Armonk, NY, USA), and Minitab, version 17, were used for analysis.

Results. During our study period, the incidence of COVID-19 positive in the pregnant population was $2.5 \%$. The mean age was 29.6 \pm 5.9 (range: $16-42$ years old). The mean body mass index (BMI) was $32 \pm 6$ and range between 17-47. The mean gestational period at diagnosis was $33.5 \pm 12.2$ weeks, and the mean GA at delivery was $38.4 \pm 1.8$ weeks.

The correlation between GA at diagnosis and GA at delivery is shown in the Figure 1. The complications by age and BMI is shown in Figures 2\&3.

In our study, none of the diagnosed pregnant population had severe/critical COVID-19 symptoms. Approximately $70.3 \%$ of the cases were asymptomatic. Symptomatic were categorized into mild types (24.3\%) which included: fever, flu symptoms, chills, body pain, weakness, alteration in taste/smell, headache, 


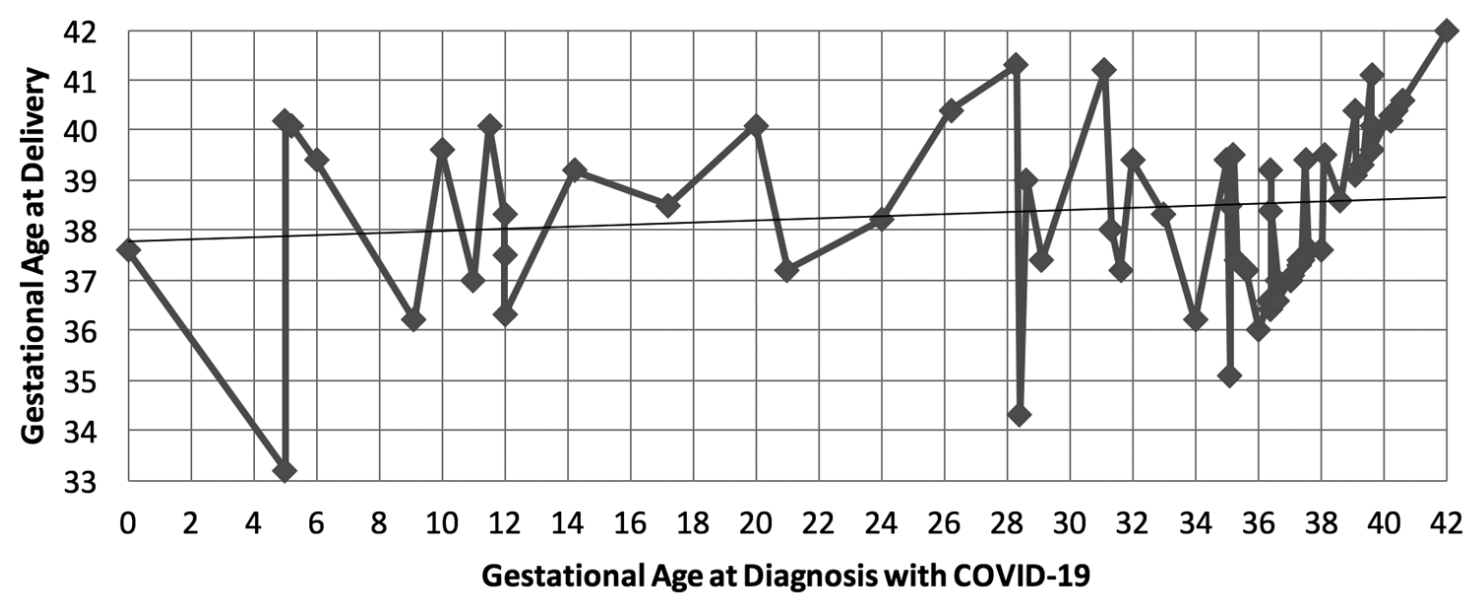

Figure 1 - Scatter plot for correlation between gestational age (GA) at diagnosis and GA at delivery. Gestational age at diagnosis and GA at delivery were not found to be significantly associated. COVID-19: coronavirus disease -19

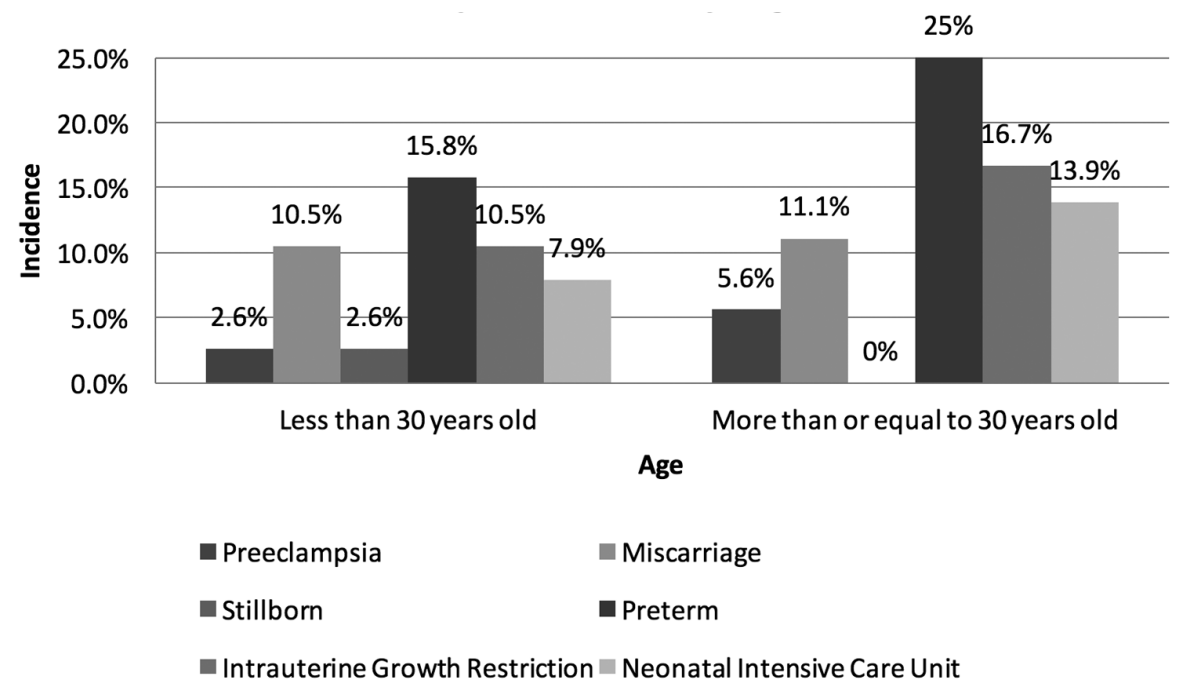

Figure 2 - Complications by age. No significant association between the complications and maternal age of coronavirus disease -19 population. $P$-values of preeclampsia $(p=0.610)$, miscarriage $(p>0.05)$, stillborn $(p>0.05)$, preterm $(p=0.310)$, intrauterine growth restriction $(p=0.510)$, neonatal intensive care unit $(p=0.468)$

upper respiratory tract symptoms, and gastrointestinal symptoms. Moderate symptoms (5.4\%) included cough with shortness of breath without desaturation and chest pain. Comorbidity analysis reported that larger number of them $(67.6 \%)$ were not known to have associated comorbidities. Approximately $32.4 \%$ of the study population had comorbidities, such as, autoimmune disorders (1.4\%), asthma (1.4\%), hypertension (5.4\%), hypothyroid (6.8\%), DM (1.4\%), and gestational DM (16.2\%).

Our population did not require any confrontational COVID-19 management. None of the 74 patients were escalated to ICU or diagnosed any morbidities and no mortalities.

Comparison of the complications in COVID-19 positive and negative groups is shown in Table 1. The obstetric outcome showed that out of the 74 pregnancies, $98.5 \%$ of babies were alive, there was one $(1.5 \%)$ stillborn and the same baby was diagnosed as congenital anomaly, and $8(10.8 \%)$ patients had miscarriage and one patient after vaginal delivery had a postpartum hemorrhage. Outcome of pregnancy by trimester at diagnosis is shown in Figure 4 and the complications by trimester at diagnosis is shown in Figure 5. 


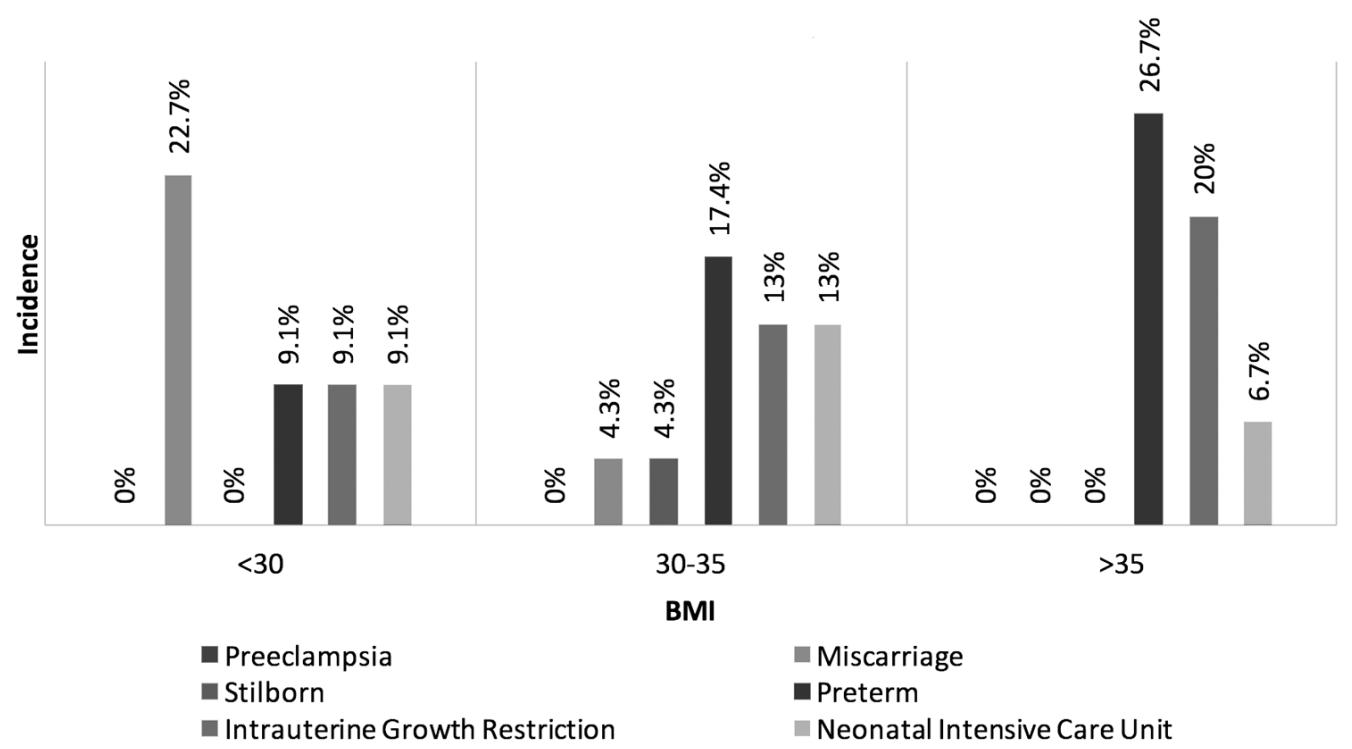

Figure 3 - Complications by body mass index (BMI). No significant association between the complications and maternal BMI of coronavirus disease -19 population. $P$-values of miscarriage $(p>0.168)$, stillborn $(p>0.05)$, preterm $(p=0.555)$, intrauterine growth restriction $(p=0.572)$, neonatal intensive care unit $(p=0.872)$

Table 1 - Comparison of complications in COVID-19 positive and negative groups.

\begin{tabular}{lcccc}
\hline Parameters & COVID-19 positive (n=74) & COVID-19 negative (N=2870) & P-value & Test \\
& \multicolumn{2}{c}{ (\%) } & & \\
\hline PET & $3(4.5)$ & $83(3.1)$ & 0.476 & Fishers exact \\
FGR & $10(15.2)$ & $56(2.1)$ & $<0.001$ & Chi-square \\
APH & $0(0)$ & $17(0.6)$ & 1.000 & Fishers exact \\
Pre-term & $15(22.7)$ & $363(13.4)$ & 0.053 & Chi-square \\
Miscarriage & $8(10.8)$ & $168(5.9)$ & 0.076 & Chi-square \\
Preterm premature rupture of membranes & $2(3.03)$ & $19(0.7)$ & 0.096 & Fishers exact \\
Stillborn & $1(1.5)$ & $20(0.7)$ & 0.415 & Fishers exact \\
PPH (EBL) & $1(1.5)$ & $24(0.9)$ & 0.472 & Fishers exact \\
NICU Admission & $8(12.1)$ & $443(16.4)$ & 0.275 & Chi-square \\
\hline
\end{tabular}

COVID-19: coronavirus disease -19, PET: pre-eclampsia, FGR: fetal growth restriction, APH: antepartum haemorrhage, PPH: postpartum haemorrhage, NICU: neonatal intensive care unit

Discussion. The COVID-19 infection in pregnancy may have harmful effects due to the partially immunocompromised state. The reference standard for the diagnosis of Corona infection is the real-time PCR. ${ }^{9}$ We used nasopharyngeal samples using PCR or SARS-CoV-2 GeneXpert in our study. The reported incidence of COVID-19 infection in pregnancy in our study was $2.5 \%$. On the other hand, a study by Nayak et $\mathrm{al}^{10}{ }^{10}$ reported that the incidence of COVID-19 infection in pregnancy was $14.4 \%$. Our study found that the mean age on identifying COVID infection was 29.6 years, with $50 \%$ of the pregnant women being in the 20-29 age group and $44.6 \%$ of them were $30-39$ years, and only $4.1 \%$ of the infected group came under the age group of 40 years and above. In contrast, Wu et al, ${ }^{11}$ summarized a report of 72,314 cases and reported that the COVID infection could occur at any age, with the older generation being the most vulnerable. In our study looking upon the complications, we did not find any significant association in relation to maternal age.

We found that COVID-19 infection was highest in pregnant women with 30-35 BMI, accounting for $38.3 \%$. It was closely followed by the BMI group less than 30 , which accounted for $36.7 \%$, and lastly, the 


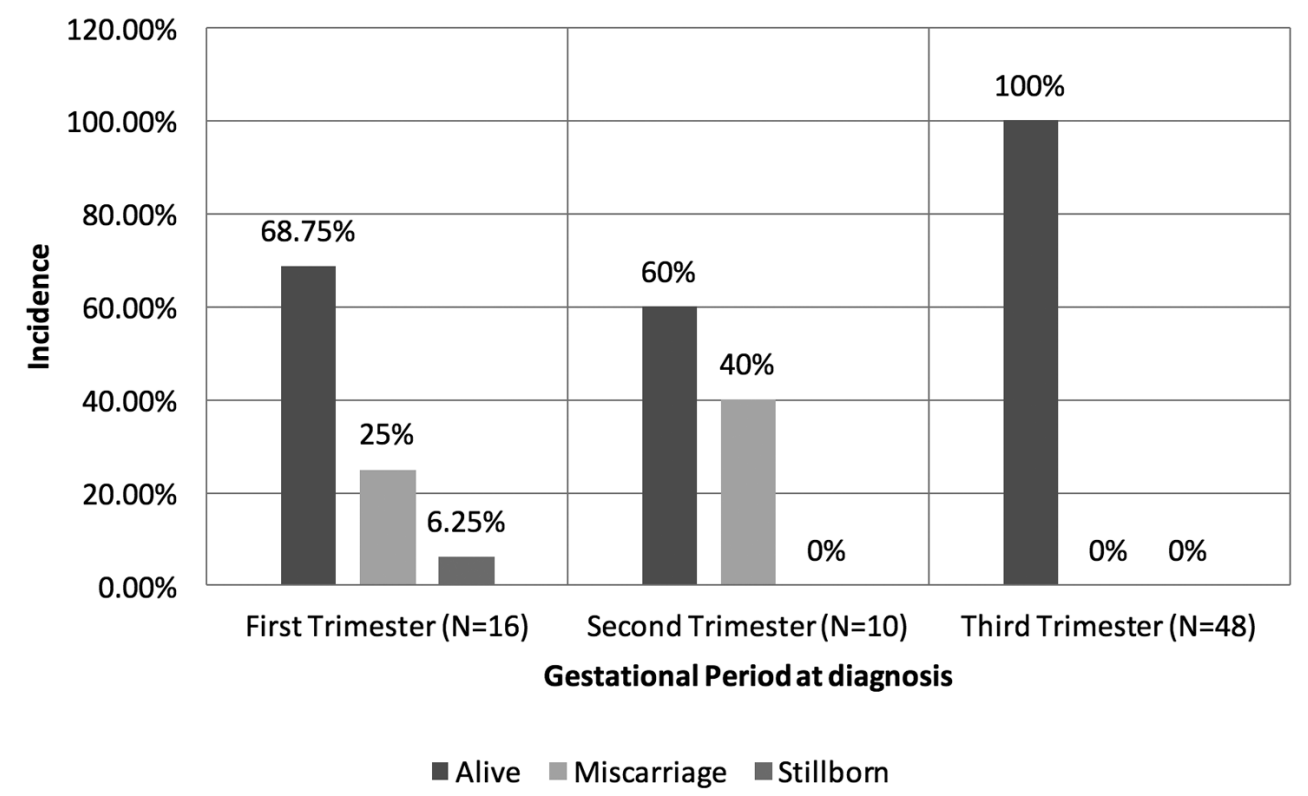

Figure 4 - Outcome of pregnancy by gestational period at diagnosis.

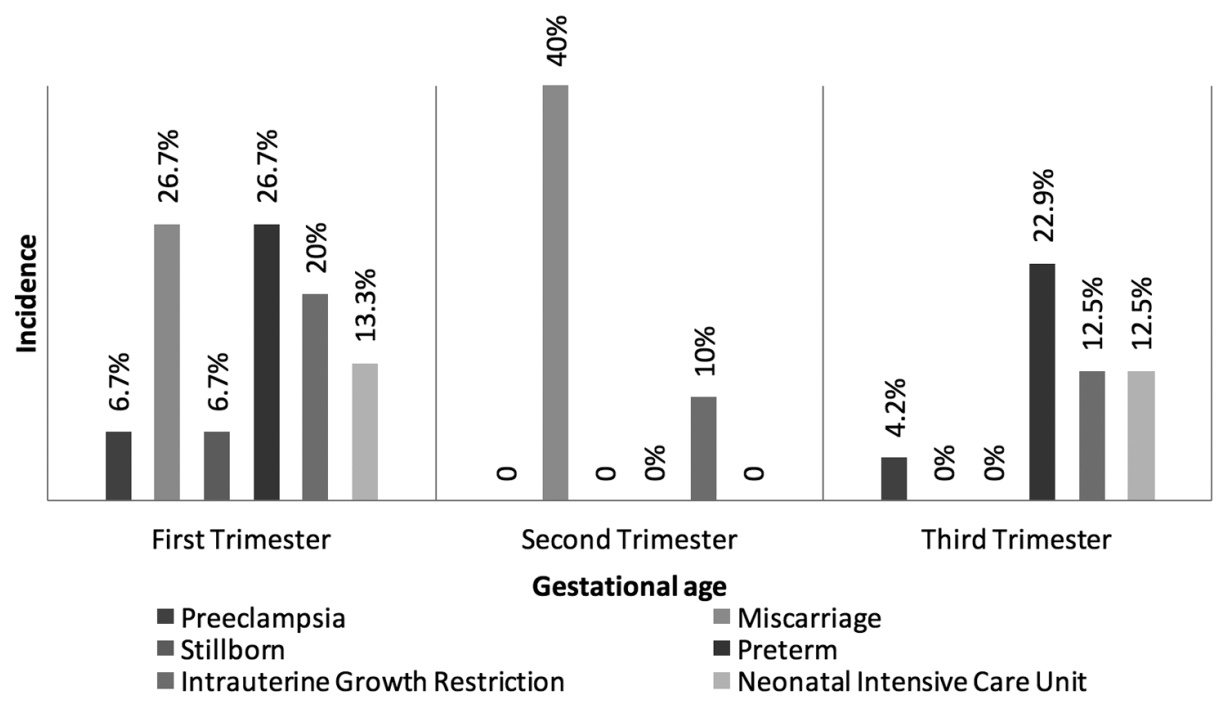

Figure 5 - Complications by gestational period at diagnosis. Significant association between miscarriage and trimester at diagnosis. $P$-values of preeclampsia $(p>0.05)$, miscarriage $(p<0.01)$, stillborn $(p=0.292)$, preterm $(p=0.300)$, intrauterine growth restriction $(p=0.877)$, neonatal intensive care unit $(p=0.703)$

BMI group of more than 35 constituted $25 \%$ of the COVID-19 infected pregnant individuals. However, we did not find any significant associations between the complications and maternal BMI. Cohort studies from Washington, United States of America, including 46 pregnant women with COVID-19 having severe diseases concluded that obesity was a major factor. ${ }^{12}$
Our study showed a high incidence during the third trimester. In contrast, a prospective cohort study by Mattar et $a l,{ }^{13}$ revealed that the mid-trimester scored the highest incidence of COVID-19, and there were no mortalities. Our study, had the same result concerning mortality. Interestingly, a Brazilian study of 114 women with SARS-CoV-2 infection found higher GA to be 
protective against the severity of the disease. ${ }^{14}$ Given the interval between GA at diagnosis and delivery, we did not find any associations.

The clinical presentation includes: fever, fatigue, dry mouth, myalgia, and shortness of breath. Few of the COVID-19 infected individuals manifested with a runny nose, sore throat, nasal congestion, hemoptysis, and even diarrhea. ${ }^{9}$ A preliminary analysis was done by Liu et al, ${ }^{15}$ on 15 pregnant women to assess the clinical features of COVID-19 infection. They deduced that fever and cough were the common symptoms. It was also concluded that all the cases were of the mild type. ${ }^{15}$

Yan et al, ${ }^{16} \mathrm{did}$ a retrospective review for 116 pregnant women from 25 hospitals in China with COVID infection and complications of respiratory illness, they found that $50.9 \%$ had fever, which was the most typical clinical presentation, next was cough (28.4\%), and asymptomatic individuals were $23.3 \%$. Our study revealed that $70.3 \%$ of the cases were asymptomatic, $24.3 \%$ had mild symptoms, and $5.4 \%$ had moderate symptoms. None of the patients had a severe or critical form of the disease. Sahin et al, ${ }^{17}$ also found that the mild type of the COVID-19 disease was found in pregnancy. Also They found that $34.5 \%$ of the confirmed COVID infected cases had comorbidities, obesity (50\%) being the most common followed by hypothyroidism which accounted for $40 \%$ of the cases. ${ }^{17}$ In our study, most of them did not have comorbidities, this could explain the mild form of the disease and the better prognosis of these individuals.

Singh et al, ${ }^{18}$ highlighted that DM might facilitate COVID-19 infection due to impaired immune response, and thereby increased viral entry. The presence of DM can determine the severity of the SARS-CoV-2 infection and can also lead to mortality. ${ }^{18}$

All of them received symptomatic and supportive management, as all of our population were mildly affected. Approximately $25.8 \%$ of them received clexane as anticoagulant in view of post caesarean section. None of them were treated with antiviral, antibiotics, or hydroxychroloquine in view of COVID-19 infection. When we looked at the treatment history of our population, we did require only symptomatic and supportive therapy as our cases are mildly affected with COVID-19 infection. Lopez et $\mathrm{al},{ }^{19}$ elaborated the treatment options which varied according to the severity of the disease. It was suggested by McIntosh, ${ }^{20}$ to refrain from the use of corticosteroids beyond 32 weeks as it may be related to increased morbidity and mortality in novel coronavirus infection. Risks versus benefits must be assessed before the administration of the drug. We administered steroids to all preterm deliveries as all of our cases were mild cases. There were no indications found to exclude the criteria for steroid administration.

According to Sahin et $\mathrm{al},{ }^{17}$ there were no admissions to the ICU. We also had a similar conclusion in our study as we did not have any morbidity.

Intensified activation of uterine natural killer cells has been implicated with spontaneous miscarriage and preeclampsia concluded by Phoswa et al. ${ }^{21}$ Preliminary findings of considering COVID-19 infection to be a risk factor for preeclampsia suggest that SARS-CoV-2 infection, despite the absent of severe respiratory symptoms, causes a pro-inflammatory state which predisposes women who are pregnant to severe preeclampsia, opined Coronado-Arroyo et al. ${ }^{22}$ In a South African study regarding maternal mortality from COVID-19 infection among pregnant women by Basu et $\mathrm{al},{ }^{23}$ hypertension increased the risk of death higher in the third trimester. The stillborn rate accounted for $66 \%$. Our study did not show any significance for preeclampsia in the COVID-19 population. However, the trimester wise outcome showed significance in miscarriage. The stillborn rate of our study was $1.5 \%$ and was associated with congenital abnormality, which might not explain the direct relation with COVID-19 infection.

In Yan et al, ${ }^{16}$ study of the 8 patients who had severe pneumonia, one had a spontaneous miscarriage and unplanned preterm delivery, constituting $6.1 \%$. Hence, they concluded that pregnant women infected with SARS-CoV-2 are not significantly related to an increased risk of unplanned preterm delivery or miscarriage. ${ }^{16}$ Mullins et $\mathrm{al},{ }^{24}$ after reviewing 21 relevant studies, inferred that preterm delivery occurred in $47 \%$ of women admitted with COVID-19 infection. According to a population-based study on the SARS-CoV-2 infection impact on pregnancy outcomes of 2225 pregnant women, they concluded that symptomatic patients had a modest increase in intrapartum fetal distress and preterm delivery. ${ }^{25}$ Our study concluded that the preterm delivery in the COVID-19 infected group was $22.7 \%$ compared to the non-infected group (13.4\%). However, it was not statistically significant.

Another complication in pregnancy concerning SARS-CoV-2 infection is IUGR. Dang et al, ${ }^{26}$ stated that the immunological changes and inadequate blood supply could be the cause for the FGR. Even though our population were with the mild form of disease, IUGR in the COVID-19 infected pregnant women were statistically significant when compared with noninfected pregnant individuals. However, the correlation may not be directly related to COVID-19. Effect of placental pathology might potentially influence the 
growth of the developing fetuses. So we recommend strict fetal surveillance for all COVID-19 infected pregnant women.

During our study, postpartum hemorrhage (PPH) was not a point for consideration, and we inferred that $1.5 \%$ of the Covid-19 positive cases had PPH. Similarly the survey by Liao et $\mathrm{al},{ }^{27}$ found no significant difference in PPH between COVID-19 infected and non-infected ones.

Newborns of COVID-19 positive mothers, admitted in neonatal intensive care unit (NICU) were found to be higher when compared to the general population deduced by Smith et $\mathrm{al}^{28}$ after evaluating 73 articles on maternal and neonatal outcomes in COVID-19 positive patients. Our study did not find any significance in NICU admissions and none of our neonates belong to the study group were infected with COVID-19 infection.

Interestingly, the overall outcome was favourable during our observation of the COVID-19 infected pregnant population. Considering the complications by trimester wise diagnosis at gestation period, the $p$-value was $<0.01$ and was statistically significant for the miscarriage. However, other associated factors for the miscarriages were not studied. So that the direct relation of COVID-19 infection cannot be included. The result of a comparative study between positive and negative groups by Nayak et $\mathrm{al}^{10}{ }^{10}$ revealed the obstetric outcome was not crucial. It was also true in the observations by Ribeiro et al, ${ }^{29}$ in which they found no neonatal asphyxia, no severe infection of COVID-19 in pregnancy and no mortalities.

Study limitations and strength. As the sample size was small the error might be possible. The reason for the small sample size could be the effective precautionary measures followed in order to reduce the transmission of the virus. Even though it was a pandemic crisis, the study was conducted in person with the positive cases. We looked at the association of GA at diagnosis and delivery. We looked at the outcome of pregnancy - trimester wise to find the significance of the COVID-19 infection in each trimester. We could identify the complications of COVID-19 infection in pregnancy, such as miscarriage and FGR and we managed the cases of FGR effectively, and we also made future recommendations in view of the COVID-19 infection in pregnancy.

Further studies are recommended to analyze the direct implications of COVID-19 in pregnant women. The population should be educated to avoid the fear of the disease as effective precautionary measures and management strategies including fetal surveillance could lead to optimal obstetric outcome. The implementation of comprehensive management plan is essential for the affected pregnant women. Accomplishing effective precautionary measures and updated protocols will help break the chain of COVID-19 transmission.

In conclusion, even though the severity of COVID-19 affected pregnant women was mild in our study, the disease had the significant association with IUGR. Complications of COVID-19 infection had no association with GA at diagnosis, maternal age, body mass index, and comorbidities. The trimester wise evaluation had the significant association for miscarriage.

Future studies of placental pathology and related etiologies will bring light to the coincidence of maternal and neonatal complications in COVID-19 infection.

Acknowledgment. The authors gratefully acknowledge the Royal Bahrain Defense Force Hospital, the Crown Prince Centre for Training and Medical Research, the National COVID-19 clinical research team, and the Maternity unit, Riffa, Bahrain. We also would like to thank Seo Content India (www.seocontentindia.in) for English language editing.

\section{References}

1. Coronavirus (COVID-19) infection and pregnancy. Royal College of Obstetricians and Gynaecologists; [Updated 2021; February 2021]. Available from: https://www.rcog.org.uk/ coronavirus-pregnancy

2. Serrano-Castro PJ, Estivill-Torrús G, Cabezudo-García P, Reyes-Bueno JA, Ciano Petersen N, Aguilar-Castillo MJ, et al. Impact of SARS-CoV-2 infection on neurodegenerative and neuropsychiatric diseases: a delayed pandemic? Neurologia (Engl Ed) 2020; 35: 245-251.

3. Akhtar H, Patel C, Abuelgasim E, Harky A. COVID-19 (SARS-CoV-2) infection in pregnancy: a systematic review. Gynecol Obstet Invest 2020; 85: 295-306.

4. Heneka MT, Golenbock D, Latz E, Morgan D, Brown R. Immediate and long-term consequences of COVID-19 infections for the development of neurological disease. Alzheimers Res Ther 2020; 12: 69.

5. Wang Y, Chen L, Wu T, Shi H, Li Q, Jiang H, et al. Impact of Covid-19 in pregnancy on mother's psychological status and infant's neurobehavioral development: a longitudinal cohort study in China. BMC Med 2020; 18: 347.

6. Elsaddig M, Khalil A. Effects of the COVID pandemic on pregnancy outcomes. Best Pract Res Clin Obstet Gynaecol 2021; 73: 125-136.

7. Novel Coronavirus 2019 (COVID-19). The American Collage of Obstetricians and Gynecologists; [Updated 2021; 8 July 2021]. Available from: https://www.acog.org/clinical/ clinical-guidance/practice-advisory/articles/2020/03/novelcoronavirus-2019

8. Ryan GA, Purandare NC, McAuliffe FM, Hod M, Purandare CN. Clinical update on COVID-19 in pregnancy: a review article. J Obstet Gynaecol Res 2020; 46: 1235-1245. 
9. Ai T, Yang Z, Hou H, Zhan C, Chen C, Lv W, et al. Correlation of chest CT and RT-PCR testing for coronavirus disease 2019 (COVID-19) in China: a report of 1014 cases. Radiology 2020; 296: E32-E40.

10. Nayak AH, Kapote DS, Fonseca M, Chavan N, Mayekar $\mathrm{R}$, Sarmalkar M, et al. Impact of the coronavirus infection in pregnancy: a preliminary study of 141 patients. $J$ Obstet Gynaecol India 2020; 70: 256-261.

11. Wu Z, McGoogan JM. Characteristics of and important lessons from the coronavirus disease 2019 (COVID-19) outbreak in China: summary of a report of 72314 cases from the Chinese Center for Disease Control and Prevention. JAMA 2020; 323: 1239-1242.

12. Lokken EM, Walker CL, Delaney S, Kachikis A, Kretzer NM, Erickson A, et al. Clinical characteristics of 46 pregnant women with a severe acute respiratory syndrome coronavirus 2 infection in Washington State. Am J Obstet Gynecol 2020; 223: 911.

13. Mattar CN, Kalimuddin S, Sadarangani SP, Tagore S, Thain S, Thoon KC, et al. Pregnancy outcomes in COVID-19: a prospective cohort study in Singapore. Ann Acad Med Singap 2020; 49: 857-869.

14. Tutiya C, Mello F, Chaccur G, Almeida C, Galvão E, Barbosa de Souza AC, et al. Risk factors for severe and critical covid-19 in pregnant women in a single center in Brazil. J Matern Fetal Neonatal Med 2021: 1-4.

15. Liu D, Li L, Wu X, Zheng D, Wang J, Yang L, et al. Pregnancy and perinatal outcomes of women with coronavirus disease (COVID-19) pneumonia: a preliminary analysis. AJR Am J Roentgenol 2020; 215: 127-132.

16. Yan J, Guo J, Fan C, Juan J, Yu X, Li J, et al. Coronavirus disease 2019 in pregnant women: a report based on 116 cases. Am J Obstet Gynecol 2020; 223: 111.

17. Sahin D, Tanacan A, Erol SA, Anuk AT, Eyi EGY, Ozgu-Erdinc AS, et al. A pandemic center's experience of managing pregnant women with COVID-19 infection in Turkey: a prospective cohort study. Int J Gynaecol Obstet 2020; 151: 74-82.

18. Singh AK, Gupta R, Ghosh A, Misra A. Diabetes in COVID-19: prevalence, pathophysiology, prognosis and practical considerations. Diabetes Metab Syndr 2020; 14: 303-310.
19. López M, Gonce A, Meler E, Plaza A, Hernández S, MartinezPortilla RJ, et al. Coronavirus disease 2019 in pregnancy: a clinical management protocol and considerations for practice. Fetal Diagn Ther 2020; 47: 519-528.

20. McIntosh JJ. Corticosteroid guidance for pregnancy during COVID-19 pandemic. Am J Perinatol 2020; 37: 809-812.

21. Phoswa WN, Naicker T, Ramsuran V, Moodley J. Preeclampsia: the role of highly active antiretroviral therapy and immune markers. Inflamm Res 2019; 68: 47-57.

22. Coronado-Arroyo JC, Concepción-Zavaleta MJ, ZavaletaGutiérrez FE, Concepción-Urteaga LA. Is COVID-19 a risk factor for severe preeclampsia? Hospital experience in a developing country. Eur J Obstet Gynecol Reprod Biol 2021; 256: 502-503.

23. Basu JK, Chauke L, Magoro T. Maternal mortality from COVID 19 among South African pregnant women. J Matern Fetal Neonatal Med 2021: 1-3.

24. Mullins E, Evans D, Viner RM, O’Brien P, Morris E. Coronavirus in pregnancy and delivery: rapid review. Ultrasound Obstet Gynecol 2020; 55: 586-592.

25. Crovetto F, Crispi F, Llurba E, Pascal R, Larroya M, Trilla C, et al. Impact of severe acute respiratory syndrome coronavirus 2 infection on pregnancy outcomes: a population-based study. Clin Infect Dis 2021; 73: 1768-1775.

26. Dang D, Wang L, Zhang C, Li Z, Wu H. Potential effects of SARS-CoV-2 infection during pregnancy on fetuses and newborns are worthy of attention. J Obstet Gynaecol Res 2020; 46: 1951-1957.

27. Liao J, He X, Gong Q, Yang L, Zhou C, Li J. Analysis of vaginal delivery outcomes among pregnant women in Wuhan, China during the COVID-19 pandemic. Int J Gynaecol Obstet 2020; 150: 53-57.

28. Smith V, Seo D, Warty R, Payne O, Salih M, Chin KL, et al. Maternal and neonatal outcomes associated with COVID-19 infection: a systematic review. PLoS One 2020; 15: e0234187.

29. Nunes Ribeiro CJ, Almeida Lima SVM, Dos Santos AD. COVID-19 and pregnancy outcomes: initial findings show little threat, but more data are needed. Evid Based Nurs 2021; 24: 59 . 